\title{
Analytical validation of the Percepta genomic sequencing classifier; an RNA next generation sequencing assay for the assessment of Lung Cancer risk of suspicious pulmonary nodules
}

Marla K. Johnson, Shuyang Wu, Daniel G. Pankratz, Grazyna Fedorowicz, Jessica Anderson, Jie Ding, Mei Wong, Manqiu Cao, Joshua Babiarz, Lori Lofaro, P. Sean Walsh, Giulia C. Kennedy and Jing Huang ${ }^{*}$ (D)

\begin{abstract}
Background: Bronchoscopy is a common procedure used for evaluation of suspicious lung nodules, but the low diagnostic sensitivity of bronchoscopy often results in inconclusive results and delays in treatment. Percepta Genomic Sequencing Classifier (GSC) was developed to assist with patient management in cases where bronchoscopy is inconclusive. Studies have shown that exposure to tobacco smoke alters gene expression in airway epithelial cells in a way that indicates an increased risk of developing lung cancer. Percepta GSC leverages this idea of a molecular "field of injury" from smoking and was developed using RNA sequencing data generated from lung bronchial brushings of the upper airway. A Percepta GSC score is calculated from an ensemble of machine learning algorithms utilizing clinical and genomic features and is used to refine a patient's risk stratification.

Methods: The objective of the analysis described and reported here is to validate the analytical performance of Percepta GSC. Analytical performance studies characterized the sensitivity of Percepta GSC test results to input RNA quantity, the potentially interfering agents of blood and genomic DNA, and the reproducibility of test results within and between processing runs and between laboratories.

Results: Varying the amount of input RNA into the assay across a nominal range had no significant impact on Percepta GSC classifier results. Bronchial brushing RNA contaminated with up to 10\% genomic DNA by nucleic acid mass also showed no significant difference on classifier results. The addition of blood RNA, a potential contaminant in the bronchial brushing sample, caused no change to classifier results at up to $11 \%$ contamination by RNA proportion. Percepta GSC scores were reproducible between runs, within runs, and between laboratories, varying within less than $4 \%$ of the total score range (standard deviation of 0.169 for scores on 4.57 scale).

(Continued on next page)
\end{abstract}

*Correspondence: jing@veracyte.com

Veracyte, Inc., South San Francisco, CA 94080, USA

(c) The Author(s). 2021 Open Access This article is licensed under a Creative Commons Attribution 4.0 International License, which permits use, sharing, adaptation, distribution and reproduction in any medium or format, as long as you give appropriate credit to the original author(s) and the source, provide a link to the Creative Commons licence, and indicate if changes were made. The images or other third party material in this article are included in the article's Creative Commons licence, unless indicated otherwise in a credit line to the material. If material is not included in the article's Creative Commons licence and your intended use is not permitted by statutory regulation or exceeds the permitted use, you will need to obtain permission directly from the copyright holder. To view a copy of this licence, visit http://creativecommons.org/licenses/by/4.0/ The Creative Commons Public Domain Dedication waiver (http://creativecommons.org/publicdomain/zero/1.0/) applies to the data made available in this article, unless otherwise stated in a credit line to the data. 
(Continued from previous page)

Conclusions: The analytical sensitivity, analytical specificity, and reproducibility of Percepta GSC laboratory results were successfully demonstrated under conditions of expected day to day variation in testing. Percepta GSC test results are analytically robust and suitable for routine clinical use.

Keywords: Percepta, Genomic sequencing classifier, Molecular diagnostic, Lung lesion, Bronchial brushing specimen, Analytical validation

\section{Background}

Lung cancer is the second most common type of cancer and the leading cause of cancer death in the United States, with 235,760 new cases and 131,880 deaths expected in 2021 [1]. Screening by low dose Computed Tomography (CT) has been shown to diagnose lung cancer at an earlier stage, resulting in improved prognosis and a relative decrease in mortality rates of $20 \%$. However, the vast majority of nodules found by chest CT are benign, resulting in a high false positive rate of around 96\% [2]. Currently, the American College of Chest Physicians (ACCP) and the National Cancer Comprehensive Network (NCCN) practice guidelines recommend an assessment of the risk of malignancy as the first step in lung nodule management. Nodules with a low risk of malignancy $(<10 \%)$ are recommended for radiological surveillance, while nodules with a high risk of malignancy (>60\%) are referred for surgical resection [3-5]. Patients with an intermediate risk of malignancy are often recommended to undergo a bronchoscopy in order to obtain a diagnosis. However, the diagnostic sensitivity of bronchoscopy varies considerably, based on a nodule's location and size [6]. Nondiagnostic bronchoscopies may result in delayed diagnosis or unnecessary invasive procedures in patients with benign nodules.

The clinical management of suspicious lung nodules may benefit from the use of genomic tests [7]. Studies have shown that exposure to tobacco smoke alters gene expression in airway epithelial cells in a way that may increase the risk of developing lung cancer [8-10]. Gene expression profiling of epithelial cells collected during bronchoscopy could thus potentially improve the sensitivity of bronchoscopy for lung cancer detection [8-10]. Based on these ideas, a microarray-based clinicalgenomic classifier, the Percepta Bronchial Genomic Classifier (BGC), was developed and validated in large, multicenter prospective trials in current and former smokers who had undergone bronchoscopy that did not return a pathology diagnosis $[11,12]$. The primary clinical utility of the Percepta BGC was to reclassify the risk of malignancy in low and intermediate pre-test risk patients to low and very low post-test risk, respectively. In a clinical utility study, patients with down-classified post-test risk were more likely to undergo surveillance rather than an invasive procedure [13].
The Percepta Genomic Sequencing Classifier (GSC) is an RNA sequencing-based clinical-genomic risk stratification algorithm with additional clinical utility beyond that of the Percepta BGC. Similar to Percepta BGC, the main utility of Percepta GSC is in the downclassification of risk in low and intermediate pre-test risk samples. In a prospective clinical validation, the Percepta GSC down-classified low and intermediate pre-test risk patients with a sensitivity of 91\% (95\% CI 81-97\%) and a specificity of $45 \%$ (95\% CI 37-53\%), resulting in a negative predictive value of $95 \%$ (95\% CI 89-98\%). The Percepta GSC can also accurately reclassify intermediate and high pre-test risk patients to high and very high post-test risk, respectively. Twelve percent of intermediate pre-test risk patients were reclassified as having high post-test risk of malignancy, with a positive predictive value of $65 \%$ (95\% CI 44-82\%). Additionally, 27\% of high pre-test risk patients were reclassified to very high posttest risk with a positive predictive value of $91 \%$ (95\% CI $78-97 \%)$ [14]. The clinical utility of up-classification of patients should be evident in decreased time to treatment or surgical resection.

Prior to use in the clinical setting, novel diagnostic tests, including genomic assays, must undergo analytical testing to show that assay results are robust and stable under the conditions expected during routine laboratory use. This process of testing variation in the technical parameters of an assay, known as analytical validation, characterizes the performance of the test in real-life settings where a variety of reagent lots, equipment, and human operators, as well the presence of common potentially interfering agents, may be encountered [15]. Accurately estimating the nominal variation in these technical parameters is an important factor in the design of analytical variation studies.

The Evaluation of Genomic Applications in Practice and Prevention (EGAPP) Working Group and the Centers for Disease Control's ACCE Project (Analytic validity, Clinical validity, Clinical utility and associated Ethical, legal and social implications) have published criteria for evaluating the analytical validity of novel genomic tests [16, 17]. Following these criteria, Percepta GSC was evaluated for variation and reproducibility of test results in response to variation in input RNA quantity, the potentially contaminating agents blood and 
genomic DNA, and intra-run, inter-run, and interlaboratory test reproducibility (Fig. 1). The results presented here demonstrate that Percepta GSC results are robust to a variety of technical variation encountered under routine laboratory testing.

The remainder of the paper is structured as follows. In the Methods section, specimen collection, laboratory workflow, and data analysis are described. In the Results section, experimental design and results of each of the analytical validation studies are described in detail. In the Discussion section, the overall robustness of Percepta GSC is discussed.

\section{Methods}

\section{Specimens}

Bronchial brushing samples were collected from patients who underwent bronchoscopy as part of the clinical management for suspicious lung nodules. Samples for this study were previously collected from patients enrolled in either the Airway Epithelial Gene Expression in the Diagnosis of Lung Cancer (AEGIS-1 and AEGIS-2) [11] study or the Percepta BGC Registry study [13]. Institutional review board (IRB) approval was obtained by each institution prior to study commencement and informed consent was obtained from all patients prior to their bronchoscopy. After sample collection, bronchial brushings were stored in a nucleic acid preservative (RNAprotect, QIAGEN, Hilden, Germany) and frozen at $-80^{\circ} \mathrm{C}$ prior to processing.

Samples used for analytical validation were identical to those encountered in the CLIA lab in terms of sample type, quantity, collection method, and experimental process.
Fresh peripheral blood samples were collected from healthy voluntary participants. Immediately after collection into blood tubes with no preservative, blood samples were mixed with RNAprotect and subsequently processed at Veracyte.

\section{RNA extraction, amplification, and sequencing}

Total RNA was extracted from each brushing sample according to the manufacturer's instructions using the miRNA easy Mini Kit (QIAGEN, Hilden, Germany). Quantification was performed using the QuantiFluor RNA System (Promega, Madison, WI), and $50 \mathrm{ng}$ of RNA was used as input to the TruSeq RNA Access Library Prep procedure (Illumina, San Diego, CA), which enriches for the coding transcriptome. Libraries meeting quality control criteria for amplification yields were sequenced using NextSeq 500 instruments $(2 \times 75$ bp paired-end reads) with the High Output Kit (Illumina, San Diego, CA).

Raw sequencing (FASTQ) files were aligned to the $\mathrm{Hu}$ man Reference assembly 37 (Genome Reference Consortium) using the STAR RNA-seq aligner software [18]. Samples and sequencing runs that met pre-specified criteria for the number and quality of reads, as well as genomic representation and read depth, were used for downstream analysis. Based on annotated Ensembl genes, uniquely mapped reads were summarized using HTSeq [19]. The sequencing data was further filtered and normalized as described in [14].

\section{Data analysis}

All data analysis was done in $\mathrm{R}$ version 3.5.0. The Percepta GSC is an ensemble of machine learning models

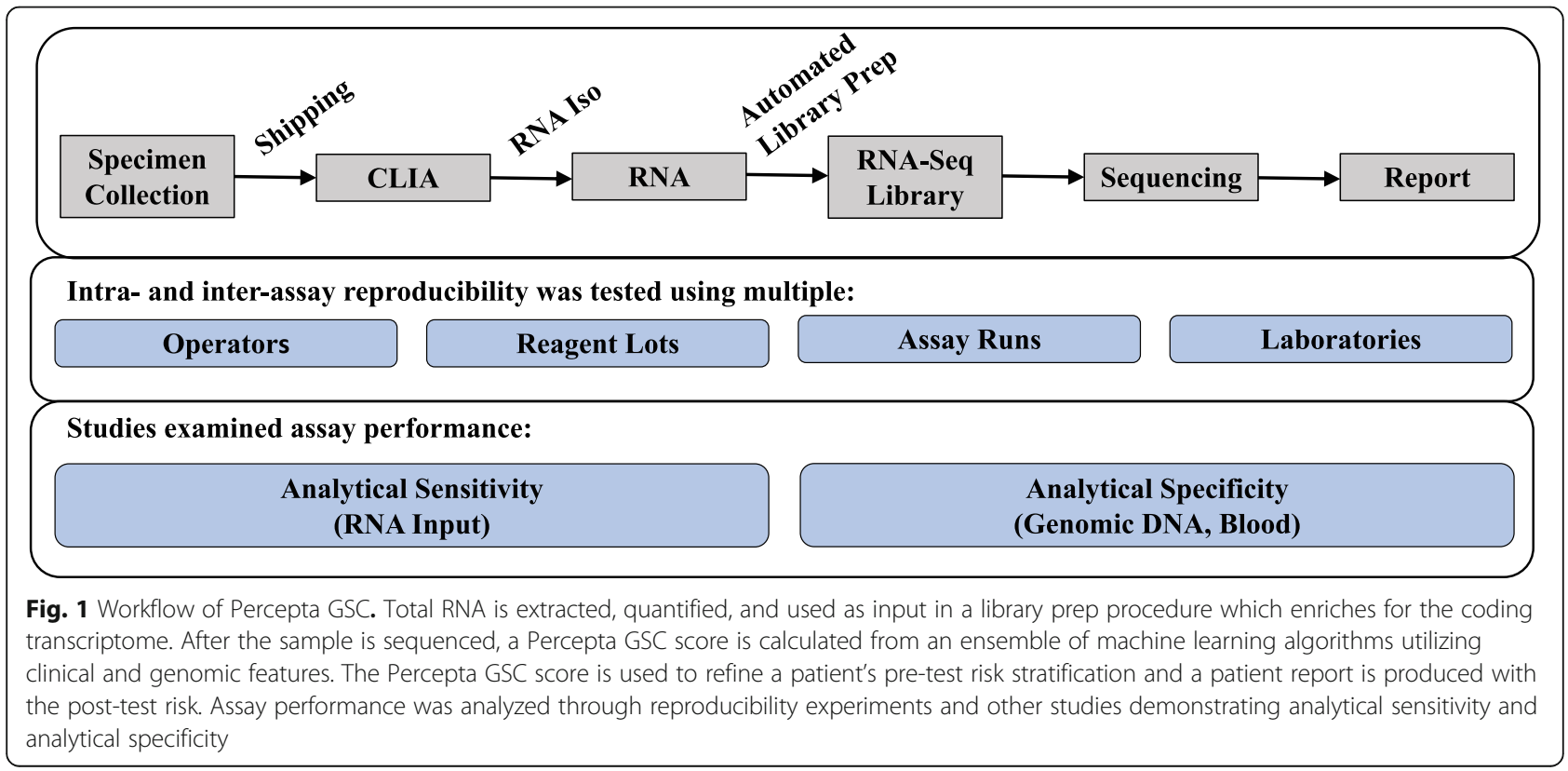


using four clinical features (age, pack year, inhaled medication usage, and specimen collection timing) and 1232 gene features as inputs [14].

Linear mixed-effect models (Eq. 1) were used to evaluate the effects of RNA input amount and interfering genomic DNA on classification scores $\left(S_{i j k}\right)$. As seen in Eq. 1, $\mu_{i}$ denoted the sample effect and was modeled as a random effect, $b_{j}$ denoted the experimental effect and was modeled as a fixed effect, $k$ denoted technical replicates, and $\varepsilon_{i j k}$ was the residual. For the total RNA input quantity analysis, the experimental effect was the RNA input amount $(10,15,20,36$, or $50 \mathrm{ng}$ RNA in one analysis and 15, 50, or $100 \mathrm{ng}$ RNA in a second analysis). For the interfering genomic DNA analysis, the experimental effect was the percentage of DNA by mass added to input RNA $(0,3,5 \%$, or $10 \%)$. For each model, analysis of variance (ANOVA) analysis tested whether the experimental effect was significant. $P$-values were considered significant at $5 \%$.

$$
S_{i j k}=\mu_{i}+b_{j}+\varepsilon_{i j k}
$$

To analyze assay reproducibility, the classifier scores were evaluated using linear mixed effect models (Eq. 2). For this equation, $\mu_{i}$ denoted the sample effect and was modeled as a fixed effect, $r_{j}$ denoted the run effect and was modeled as a random effect, and $\mu_{i}: r_{j}$ denoted the interaction between sample $i$ and run $j$ and was modeled as a random effect.

$$
S_{i j k}=\mu_{i}+r_{j}+\mu_{i}: r_{j}+\varepsilon_{i j k}
$$

To analyze inter-laboratory accuracy, the classifier scores $\left(S_{i j}\right)$ were evaluated using a linear model (Eq. 3). In this model, $\mu_{i}$ denoted the sample effect, $k$ denoted technical replicates, and $\varepsilon_{i k}$ was the residual.

$$
S_{i k}=\mu_{i}+\varepsilon_{i k}
$$

All 95\% confidence intervals (CI) for standard deviations (SD) were obtained by bootstrap where the residuals of the linear model or linear mixed-effect model were sampled with replacement.

A simulation study was performed in order to assess the degree of technical noise which could be added to classifier scores before the performance of the classifier would be severely affected. In the first step of each simulation, random score noise was generated from a normal distribution with a mean of 0 and SD varying between 0.01 and 10. This random noise was added to the Percepta GSC validation scores to create simulated scores. In the second step of each simulation, performance metrics were calculated for the simulated scores, including sensitivity, specificity, positive predictive value (PPV), and negative predictive value (NPV). The percentage of patients whose cancer risk was either increased or decreased by Percepta GSC testing was also calculated. Additionally, the flip-rate, or the percentage of altered classifier calls which occurred due to the added noise, was calculated at each noise level. The two steps described here were repeated 1000 times for each noise level, and median performance metrics were computed across all simulations at each noise level. The maximum allowable score variability was determined by choosing the largest variability for which all the performance metrics continued to meet product requirements.

\section{Results}

Analytical sensitivity - total RNA input quantity

The Percepta GSC test specifies that $50 \mathrm{ng}$ of total RNA be used as input to the library preparation procedure, though the actual input amount may vary due to nominal quantitation measurement error (coefficients of variation (CV) of technical replicates in a single quantitation batch of up to 20\%, and batch-to-batch CVs of up to $30 \%$, data not shown) or pipetting (precision and accuracy $1 \%$ of volume typically). The sensitivity of the Percepta GSC to lower total RNA input amounts, which have the potential to result in less diverse library populations that might occur under conditions of measurement error, was evaluated. Four total RNA samples covering the Percepta GSC score space were plated in triplicate using input amounts of 10, 15, 20, 36, and 50 ng RNA, and the Percepta GSC score was determined for each run (Fig. 2). Percepta GSC scores the brushing samples were not significantly different for the lowered RNA input amounts, when evaluated with a linear mixed effect model ( $p$-value $=0.07)$, though there was a trend towards lower Percepta GSC scores in the smallest input amount (10 ng RNA). In a separate study, 15 samples were each run once using input amounts of 15, 50 and $100 \mathrm{ng}$ RNA, and the Percepta GSC scores were determined for each run (Fig. 2). Percepta GSC scores did not differ significantly with different RNA input amounts, when evaluated with a linear mixed effect model $(p$-value $=0.22$ ). Percepta GSC test results are robust over a range beyond that which would be expected under routine test conditions, thus laboratory sample measurement error has an insignificant impact to test results even under conditions of RNA input 5-fold lower than nominal levels.

\section{Analytical specificity - blood}

Bronchial brushing samples may contain small amounts of blood due to the procedure used to collect bronchial epithelial cells, and blood could potentially have an impact on the genomic signal [20]. The maximum theoretical blood contamination was estimated to be approximately $7.5 \%$, based on the volume of blood that could be captured in the brush and sheath and the 


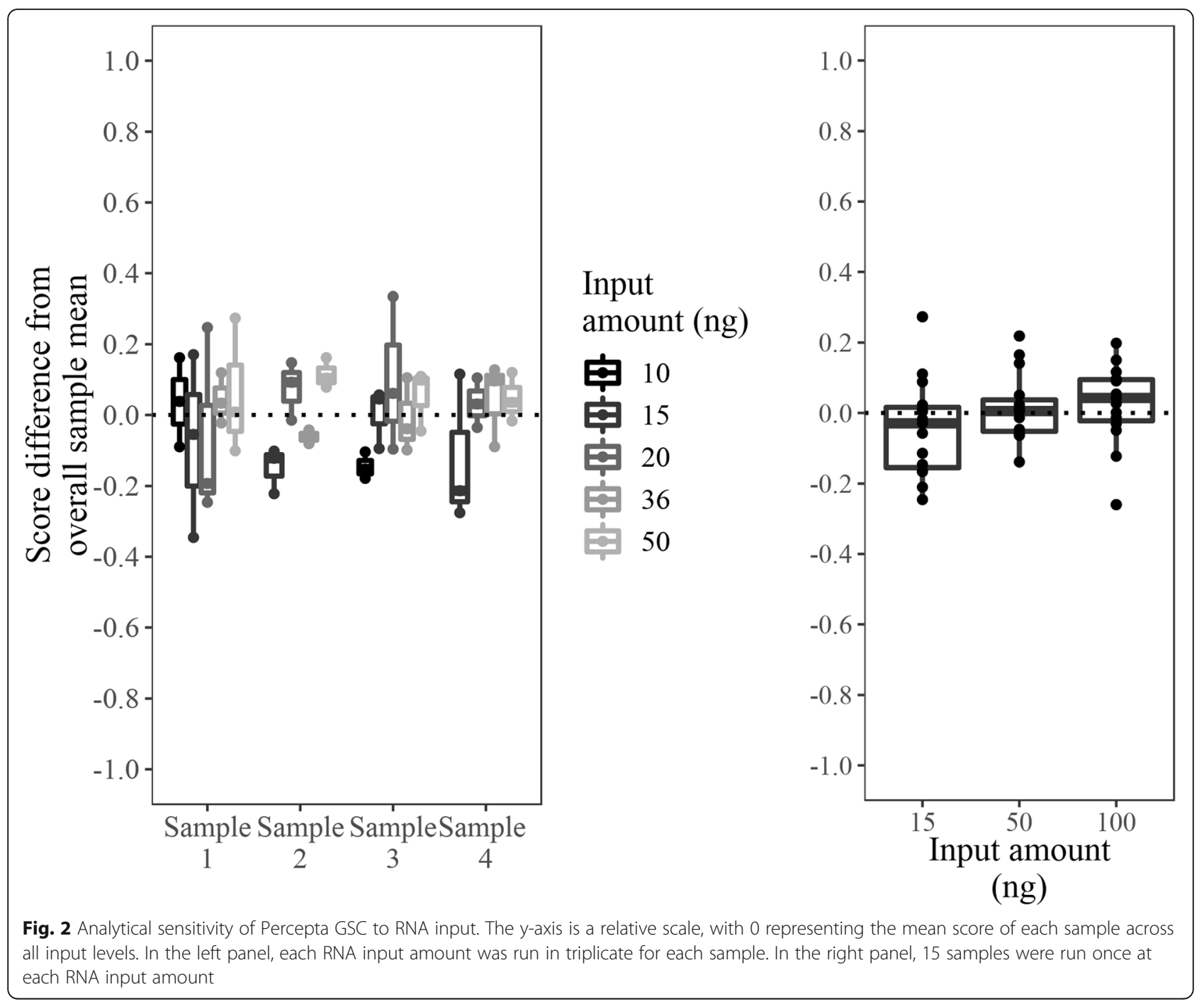

lowest acceptable RNA recovery [21]. Analysis of blood contamination levels in the total RNA from bronchial brush samples showed that less than $1 \%$ of the clinical samples had more than $1 \%$ of blood derived RNA, with the most extreme samples having around 10\% of blood derived RNA [21]. In order to evaluate the potential of blood as an interfering substance on Percepta GSC test, whole blood total RNA from two donors was added to total RNA purified from three bronchial brushing samples. The three bronchial brushing derived RNA samples were chosen to represent the three pre-test risk groups that Percepta GSC patients are placed into by their physician prior to bronchoscopy: low (malignancy risk $<10 \%$ ), intermediate (malignancy risk $10-60 \%$ ), and high (malignancy risk $>60 \%$ ). Pure bloodderived total RNA samples score high (malignant) in Percepta GSC score space and would theoretically increase the score for a bronchial sample when mixed. Blood samples were chosen from both the lower end and higher end of the score space seen in blood-derived total RNA samples.
Each unadulterated bronchial brushing RNA sample and blood RNA sample was run in triplicate. The two blood RNA samples were added to the three bronchial brushing derived RNA samples at 5, 10, 20, 50, and 75\% blood RNA, while maintaining the total RNA input to the test constant at $50 \mathrm{ng}$. Samples admixed at the lower three proportions $(5,10,20 \%)$ were run in triplicate, while samples admixed at higher proportions $(50,75 \%)$ were run in duplicate. Percepta GSC scores were generated for all mixtures and the best fit curve was plotted through the scores. For each bronchial sample, the percentage of added blood that would alter their original Percepta GSC was estimated from the curve (Table 1, Supplemental Figure 1).

With no blood RNA added, the low pre-test risk sample was reclassified to very low post-test risk. The patient's Percepta GSC call remained unchanged compared to the pre-test risk when the amount of added blood RNA is $25 \%$ from the higher scoring blood sample or 
Table 1 Percentage of interfering blood required to alter Percepta GSC call

\begin{tabular}{|c|c|c|c|}
\hline Pre-Test Risk & Post-Test Risk & Low Malignant Blood & High Malignant Blood \\
\hline \multirow[t]{2}{*}{ Low } & Very Low & $<28 \%$ & $<25 \%$ \\
\hline & Low & $>28 \%$ & $>25 \%$ \\
\hline \multirow[t]{3}{*}{ Intermediate } & Low & $<20 \%$ & $<11 \%$ \\
\hline & Intermediate & $>20 \%$ & $11-97 \%$ \\
\hline & High & No call change & $>97 \%$ \\
\hline High & Very High & No call change & No call change \\
\hline
\end{tabular}

$28 \%$ from the lower scoring blood sample. With no blood RNA added, the intermediate pre-test risk sample was reclassified to a post-test risk of low risk. The patient's Percepta GSC call remained unchanged compared to pre-test risk when the amount of added blood RNA is $11 \%$ from the higher scoring blood sample or $20 \%$ from the lower scoring blood sample. Additionally, the sample would classify as high risk at a contamination of $97 \%$ of the higher scoring blood sample. The sample did not classify as high risk at any level of contamination with the lower scoring blood sample. The pre-test high risk sample remained high post-test under all blood mixture conditions tested. Since the maximum blood content seen in patient samples was around $10 \%$, it is unlikely that any patient calls would be expected to be influenced or changed by the presence of nominal blood contamination. Only levels of blood contamination substantially higher than thus far observed in patient samples might disrupt the accurate reclassification of samples with intermediate or low pre-test risk to low or very low post-test risk, respectively. Under these rare conditions, the utility of the test to reduce unnecessary procedures through accurate risk reduction would potentially be impacted.

\section{Analytical specificity - genomic DNA}

Genomic DNA is a potential contaminant and interfering substance in any biological sample, as small amounts may be co-extracted with the total RNA. In clinical samples collected as part of the AEGIS-1 and AEGIS-2 studies, the amount of genomic DNA found in the processed RNA samples was consistently $1 \%$ or less [17]. In order to test the impact of genomic DNA as a possible contaminating agent, genomic DNA was added to bronchial brushing RNA samples at $0,3,5$, and $10 \%$ contamination by mass of nucleic acid, while maintaining the total RNA input to the test constant at $50 \mathrm{ng}$. For each amount of contamination, two samples were run in triplicate at 0,3 , and 5\% genomic DNA and run in quadruplicate at $10 \%$ genomic DNA. The samples were chosen to cover Percepta GSC score space, with one high-risk scoring and one low-risk scoring sample chosen to identify any potential score shift downwards or upwards towards an intermediate post-test result, respectively. A similar analytic result (a score shift) could be detected using samples of intermediate post-test results. The Percepta GSC scores of samples with gDNA additions were not significantly different than the corresponding pure RNA samples, when evaluated with a linear mixed effect model ( $p$-value $=0.49$ ) (Fig. 3 ), with no consistent trend in scores observed. Therefore, Percepta GSC results are robust in the presence of DNA contamination 10-fold higher than has been observed in clinical bronchial brush samples. DNA contamination of test RNA thus has no meaningful impact to test accuracy or clinical utility.

\section{Assay reproducibility}

In order to analyze the intra-run and inter-run reproducibility of the Percepta GSC, 33 different samples, including six control samples, were processed in triplicate across three different experimental runs in a single laboratory. For each run, the reagent lots, operators, and equipment were varied, to represent the normal variation anticipated in routine processing (Supplemental Table 1). The 27 non-control samples were chosen to represent the entire classification score space of Percepta GSC, evenly divided between high, medium, and low scoring samples. Inter-run, intra-run, and total score SDs were estimated using a mixed linear effect model. The total SD was estimated to be 0.179 (95\% CI 0.169 to 0.192; Fig. 4), with the intra-run SD estimated to be 0.117 (95\% CI 0.104 to 0.129; Fig. 4) and the inter-run SD estimated to be 0.136 (95\% CI 0.117 to 0.151 ; Fig. 4). This can be compared to the inter-class score variability between benign and malignant samples, which is almost four-fold higher (Fig. 4). The total SD was compared to the score range in the training set using cross-validation, which was calculated to be 4.57 . The total SD ascribed to technical variation therefore represents $3.9 \%$ of the total Percepta GSC score range. This amount of technical variation is similar to that seen in other commercial genomic classifiers (Supplemental Table 2). Thus, the technical variation within and between runs is far lower than the inherent biological signal on which the test operates.

To establish the impact of technical variability to the accuracy of the Percepta GSC, a simulation of added 


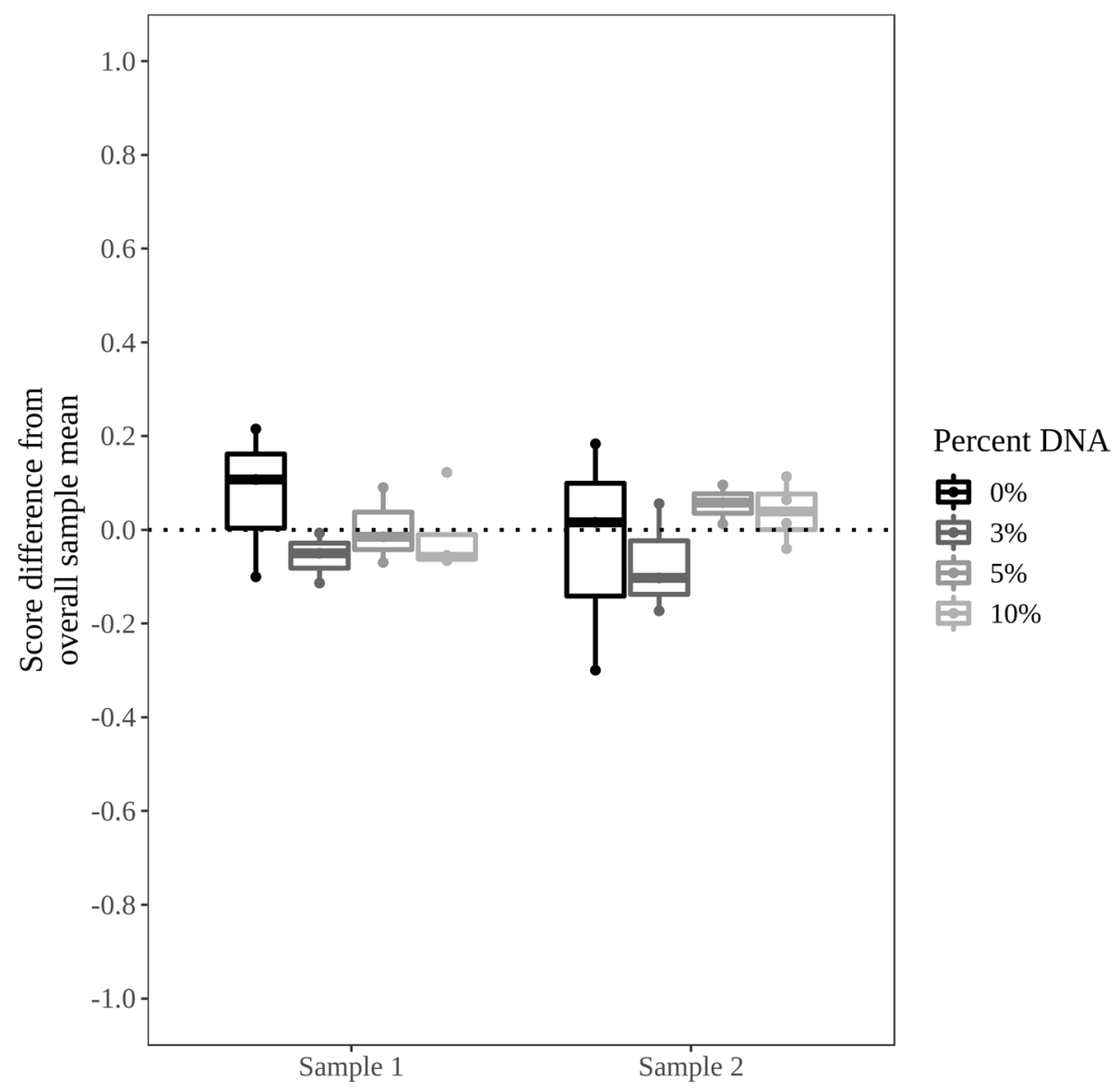

Fig. 3 Analytical specificity of Percepta GSC against genomic DNA. The $y$-axis is a relative scale, with 0 representing the mean score of each sample across all input levels. Each input amount was run in triplicate at 0,3, and 5\% genomic DNA and run in quadruplicate at 10\% genomic DNA. For each sample, the variation from the overall mean is plotted for each differing percentage of genomic DNA contamination

variability was performed in silico using 1000 simulations. Acceptable performance criteria were established for each classifier for sensitivity, specificity, NPV, and PPV, and the smallest variability at which the median performance fell below the acceptable value was determined. As technical variability was added in this simulation, the first observed effect was an increased the likelihood of a high post-test risk result for patients with intermediate pre-test risk, potentially impacting the accuracy of positive test calls. The limiting amount of variability that could be tolerated before the PPV fell below the required $50 \%$ level was 0.334 , which is $7.3 \%$ of the total Percepta GSC score range. This is two-fold higher than the observed variability in the reproducibility study.

\section{Inter-laboratory accuracy}

In order to establish the lab-to-lab accuracy of Percepta GSC, the assay was performed independently in two different laboratories: the Veracyte R\&D laboratory in which the test was developed and the Veracyte reference CLIA laboratory where patient testing will be performed (Supplemental Table 1). These physically separate laboratories are operated by different personnel using different equipment. Ninety-five samples, including six control samples, were selected in order to cover the entire score space of Percepta GSC. Total SD was estimated using a linear model to be 0.169 (95\% CI 0.146 to 0.192; Fig. 4), which is in line with the run to run, within lab variation observed in the reproducibility study. This suggests that technical variation associated with lab, equipment and operator variation is within the range of normal processing run variation in the same lab. The Percepta GSC scores generated between the two laboratories showed high correlation $\left(R^{2}=0.98\right)$. Between the two laboratories, discordant calls were made in 13 out of 131 (9.9\%) classifier calls, lower than the pre-specified acceptance criteria of $15 \%$. The discordant calls are due to normal run to run score variation in samples with scores within approximately $1 \mathrm{SD}$ of the decision boundary. 


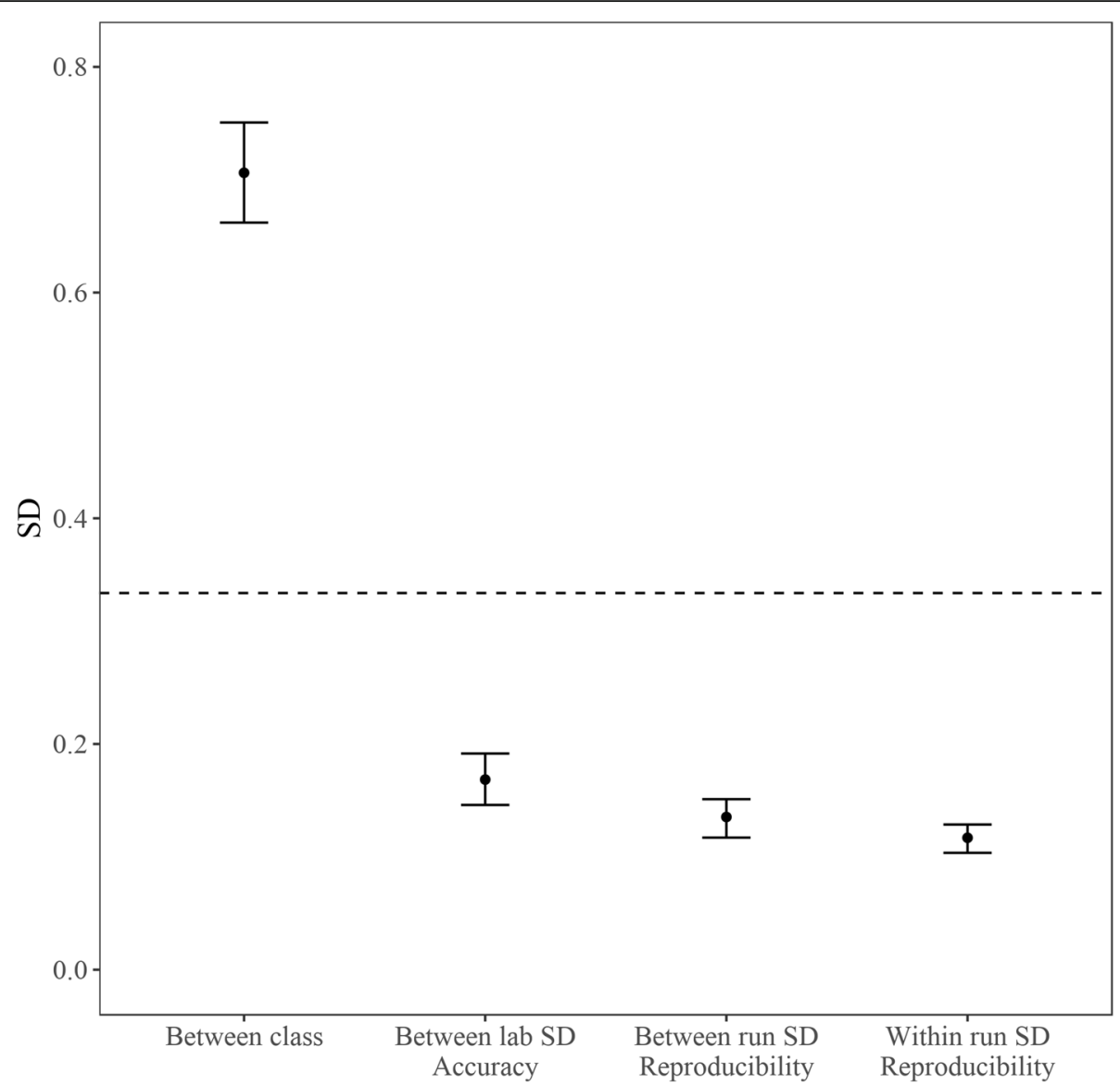

Fig. 4 Comparison of Percepta GSC score variability. The inter-class score SD includes biological variation between cancer and benign samples and was computed from samples in the Percepta GSC validation set. The dashed line denotes the SD of 0.334 , which was determined by flip-rate analysis to be the minimum SD at which performance of Percepta GSC was severely affected

\section{Discussion}

Novel genomic tests must be characterized with respect to both clinical and analytical performance before they are put into clinical use. Potential sources of technical variation, including the entire process of sample collection, storage, shipping, handling, and laboratory processing, must be evaluated for possible impact to test results $[22,23]$. Bronchial brushing specimen stability under routine collection conditions was established in the Percepta BGC analytical validation study [21]. The process described for Percepta BGC remains unchanged for clinical samples received in the Veracyte CLIA-certified laboratory for Percepta GSC testing. As such, this study was focused on analytical validation of the Percepta GSC classifier, using the new next-generation sequencing platform.

The Percepta GSC assay was found to be robust to variation in amount of RNA used in the assay and the potentially interfering substances blood and genomic DNA, with no significant difference in Percepta GSC scores noted under the conditions tested. A small impact on test performance was detected only at the extremes of RNA input (5-fold lower than nominal) or blood contamination higher than identified thus far in any bronchial brush sample. Test performance was not impacted by genomic DNA contamination at a level 10-fold higher than has been observed in clinical bronchial brush samples. To assess the impact of technical variation due to processing runs within the same laboratory versus testing done in different laboratories, Percepta GSC scores from the research laboratory where the test was developed were compared to scores generated from RNA from the same patient samples in the CLIA-certified laboratory which will run patient samples. This was in turn compared to multiple runs of the same samples within the CLIA laboratory which varied by reagent lot, equipment, day, and operator. The inter-laboratory SD of Percepta GSC scores was estimated to be $3.7 \%$ of the Percepta GSC score range, a value on par with the total SD of Percepta GSC scores within and across multiple processing runs of $3.9 \%$ of the Percepta GSC score range. 
Taken together, these studies demonstrate EGAPP level I internal validity criteria for reproducibility of test results under conditions of varying operator, equipment, and process runs [16]. These results support that the Percepta GSC is a robust and stable classifier able to confidently return clinical results under routine operation in a clinical lab.

\section{Conclusions}

Percepta GSC test results have demonstrated robustness across a variety of technical variables which may be potentially encountered as part of clinical sample testing. Use of Percepta GSC for risk stratification of suspicious lung nodules can be performed with high confidence in the clinical setting.

\section{Abbreviations}

GSC: Genomic sequencing classifier; BGC: Bronchial genomic classifier; RNA: Ribonucleic acid; DNA: Deoxyribonucleic acid; EGAPP: Evaluation of genomic applications in practice and prevention; ACCE: Analytic validity, clinical validity, clinical utility and associated ethical, legal and social implications; SD: Standard deviation; Cl: Confidence interval; PPV: Positive predictive value; NPV: Negative predictive value; CV: Coefficients of variation; CLIA: Clinical laboratory improvement amendments

\section{Supplementary Information}

The online version contains supplementary material available at https://doi. org/10.1186/s12885-021-08130-x

\section{Additional file 1.}

\section{Acknowledgements}

We would like to thank Jianchang (Jeff) Ning and Xinwu Yang for database and sequencing pipeline development and support and Jonathan Wilde and Jennifer Tom for helpful discussions and suggestions.

\section{Authors' contributions}

$D P, J D, J B, P W, G K$, and JH conceived and designed the study. DP, GF, JA, $M W, M C, J B$, and $P W$ designed and performed the sequencing experiments. MJ, SW, DP, GF, JA, JD, MW, MC, JB, PW, GK, and JH analyzed and interpreted the study. $\mathrm{LL}$ coordinated the clinical sample collection as part of the clinical validation studies. MJ, SW, and JH implemented the analysis, prepared figures, and drafted the manuscript. MJ, SW, DP, JB, PW, GK, and JH edited and revised the manuscript. All authors have read and approved the final manuscript.

\section{Funding}

Funding for this study and the publication of this article was provided by Veracyte Inc. Veracyte Inc. drafted the study design, oversaw the data analysis and manuscript preparation, and approved the decision to publish.

\section{Availability of data and materials}

The datasets generated during the current study are not publicly available due to concerns regarding patient confidentiality and proprietary information but are available upon reasonable request from the corresponding author.

\section{Declarations}

\section{Ethics approval and consent to participate}

The Percepta Registry and AEGIS cohorts were multi-center prospective studies that included patients with lung nodules who underwent clinically indicated diagnostic bronchoscopy at participating medical centers across the US, Canada and Europe. Ethics review and approval (IRB/REB/IEC) was obtained by each institution before enrollment and informed consent was obtained from all patients. A list of the committee names and approval numbers for each AEGIS-1, AEGIS-2, and Percepta BGC Registry site are included as Supplemental Table 3. All freshly collected blood samples were collected with IRB approval obtained from Liberty IRB (\#10.12.0009) and written informed consent from all participants.

\section{Consent for publication}

Not applicable.

\section{Competing interests}

All authors are employed by Veracyte Inc.

Received: 8 October 2020 Accepted: 30 March 2021

Published online: 13 April 2021

\section{References}

1. American Cancer Society. Cancer Facts \& Figures 2021. Atlanta: American Cancer Society; 2020. Available at: https://www.cancer.org/research/cancerfacts-statistics/all-cancer-facts-figures/cancer-facts-figures-2021.html

2. National Lung Screening Trial Research Team, Aberle DR, Adams AM. Reduced lung-cancer mortality with low-dose computed tomographic screening. N Engl J Med. 2011;365(5):395-409. https://doi.org/10.1056/ NEJMoa1102873.

3. Gould MK, Donington J, Lynch WR, et al. Evaluation of individuals with pulmonary nodules: when is it lung cancer? Diagnosis and management of lung cancer, 3rd ed: American College of Chest Physicians evidence-based clinical practice guidelines. Chest. 2013;143(5_suppl):e93S-120S.

4. National Comprehensive Cancer Network: Lung Cancer Screening v2.2014. NCCN Clin- ical Practice Guidelines in Oncology (NCCN Guidelines). Washington, PA: National Comprehensive Cancer Network; 2014 Available at: http://www.nccn.org/professionals/physician_gls/pdf/lung_screening.pdf. Accessed 1 June 2020

5. Baldwin DR, Callister ME. Guideline development group. The British Thoracic Society guidelines on the investigation and management of pulmonary nodules. Thorax. 2015;70(8):794-8. https://doi.org/10.1136/thoraxjnl-2015-2 07221.

6. Rivera MP, Mehta AC, Wahidi MM. Establishing the diagnosis of lung cancer: diagnosis and management of lung cancer, 3rd ed: American College of Chest Physicians evidence based clinical practice guidelines. Chest. 2013: 143(5 Suppl):e142S-65S. https://doi.org/10.1378/chest.12-2353.

7. Kosvyra A, Maramis C, Chouvarda I. Developing an integrated genomic profile for Cancer patients with the use of NGS data. Emerg Sci J. 2019;3(3): 157-67. https://doi.org/10.28991/esj-2019-01178.

8. Spira A, Beane J, Shah V, Liu G, Schembri F, Yang X, et al. Effects of cigarette smoke on the human airway epithelia cell transcriptome. Proc Natl Acad Sci U S A. 2004:101(27):10143-8. https://doi.org/10.1073/pnas.0401422101.

9. Wistuba II, Mao L, Gazdar AF. Smoking molecular damage in bronchial epithelium. Oncogene. 2002;21(48):7298-306. https://doi.org/10.1038/sj. onc.1205806.

10. Spira A, Beane JE, Shah V, Sterling K, Liu G, Schembri F, et al. Airway epithelial gene expression in the diagnostic evaluation of smokers with suspect lung cancer. Nat Med. 2007;13(3):361-6. https://doi.org/10.1038/ nm1556.

11. Silvestri GA, Vachani A, Whitney D, Elashoff M, Porta Smith K, Ferguson JS, et al. A bronchial genomic classifier for the diagnostic evaluation of lung cancer. N Engl J Med. 2015;373(3):243-51. https://doi.org/10.1056/NEJMoa1 504601.

12. Whitney DH, Elashoff MR, Porta-Smith K, Gower AC, Vachani A, Ferguson JS, Silvestri GA, Brody JS, Lenburg ME, Spira A. Derivation of a bronchial genomic classifier to lung cancer in a prospective study of patients undergoing diagnostic bronchoscopy. BMC Med Genet. 2015:8:18.

13. Lee HJ, Mazzone P, Feller-Kopman D, Yarmus L, Hogarth K, Lofaro LJ, et al. Percepta registry investigators. Impact of the Percepta genomic classifier on clinical management decisions in a multicenter prospective study. CHEST. 2021;159(1):401-12. https://doi.org/10.1016/j.chest.2020.07.067.

14. Choi Y, Qu J, Wu S, Hao Y, Zhang J, Ning J, et al. Improving Lung Cancer risk stratification leveraging whole Transcriptome RNA sequencing and machine learning across multiple cohorts. BMC Med Genet. 2020;13(Suppl 10):151. https://doi.org/10.1186/s12920-020-00782-1. 
15. Choi Y, Huang J. Validation of Genomic-Based Assay in Fang L, Su Cheng, ed. Statistical Methods in Biomarker and Early Clinical Development, Springer Nature, Switzerland, p. 117-136.

16. Teutsch SM, Bradley LA, Palomaki GE, Haddow JE, Peper M, Calonge N, et al. The evaluation of genomic applications in practice and Prevation (EGAPP) initiative: methods of the EGAPP working group. Genet Med. 2009;11(1):314. https://doi.org/10.1097/GIM.0b013e318184137c.

17. Sun F, Bruening W, Uhl S, Ballard R, Tipton R, Schoelles K. Quality, regulation, and clinical utility of laboratory-developed molecular tests. Agency for Healthcare Research and Quality, Technology Assessment Program; 2010.

18. Dobin A, Davis CA, Schlesinger F, Drenkow J, Zaleski C, Jha S, et al. STAR: ultrafast universal RNA-seq aligner. Bioinformatics. 2013;29(1):15-21. https:// doi.org/10.1093/bioinformatics/bts635.

19. Anders S, Pyl PT, Huber W. HTSeq - a Python framework to work with highthroughput sequencing data. Bioinformatics. 2015;31(2):166-9. https://doi. org/10.1093/bioinformatics/btu638.

20. Nicoleau S, Wojciak-Stothard B. Beyond thrombosis: the role of platelets in pulmonary hypertension. SciMed J. 2020;2(4):243-71. https://doi.org/10.2 8991/SciMedJ-2020-0204-7.

21. Hu Z, Whitney D, Anderson JR, Cao M, Ho C, Choi Y, et al. Analytical performance of a bronchial genomic classifier. BMC Cancer. 2016;16:61.

22. Khorasghani MA, Nejad PM, Bashi MMM, Hedayati M. Evaluation of mir-377$3 p$ expression in patients with multiple sclerosis. SciMed J. 2019;1 (2):48-54. https://doi.org/10.28991/SciMedJ-2019-0102-2.

23. Abdelaal AM, Attalla EM. Elshemey. Estimation of out-of-field dose variation using Markus ionization chamber detector. SciMed J. 2020;2(1):8-15. https:// doi.org/10.28991/SciMedJ-2020-0201-2.

\section{Publisher's Note}

Springer Nature remains neutral with regard to jurisdictional claims in published maps and institutional affiliations.

Ready to submit your research? Choose BMC and benefit from:

- fast, convenient online submission

- thorough peer review by experienced researchers in your field

- rapid publication on acceptance

- support for research data, including large and complex data types

- gold Open Access which fosters wider collaboration and increased citations

- maximum visibility for your research: over $100 \mathrm{M}$ website views per year

At BMC, research is always in progress.

Learn more biomedcentral.com/submissions 